Article

\title{
Caputo Fractional Differential Equations with Non-Instantaneous Random Erlang Distributed Impulses
}

\author{
Snezhana Hristova *(D) and Krasimira Ivanova \\ Department of Applied Mathematics and Modeling, University of Plovdiv "Paisii Hilendarski", \\ Plovdiv 4000, Bulgaria; krasbr@gmail.com \\ * Correspondence: snehri@gmail.com
}

Received: 2 May 2019; Accepted: 14 May 2019; Published: 18 May 2019

check for updates

\begin{abstract}
The $p$-moment exponential stability of non-instantaneous impulsive Caputo fractional differential equations is studied. The impulses occur at random moments and their action continues on finite time intervals with initially given lengths. The time between two consecutive moments of impulses is the Erlang distributed random variable. The study is based on Lyapunov functions. The fractional Dini derivatives are applied.
\end{abstract}

Keywords: impulsive fractional differential equations; random moments of impulses; non-instantaneous impulses; Erlang distribution; $p$-moment exponential stability

\section{Introduction}

Fractional differential equations are an adequate model of many physical phenomena that have memory and genetic characteristics [1-4]. At the same time, many evolution processes are characterized by the states' abrupt changes. These changes are adequately modeled by so-called impulses. There are two types of impulses: instantaneous impulses (their duration is negligible small) and non-instantaneous impulses (they start at some points and continue to be active on finite time intervals). Note the theory of instantaneous impulsive differential equation-known as impulsive differential equations-is well developed. At the same time, the theory of differential equations with non-instantaneous impulses is still at its initial stage (see, for example, Reference [5-11]). In many real world phenomena, the studied process has abrupt changes at random moments of time. In this case, the adequate model is, by differential equation, subject to random impulses. Impulsive differential equations with random impulsive moments differ from the study of stochastic differential equations with impulses (see, for example, Reference [12-16]). Some stability properties of differential equations with non-instantaneous impulses, starting at randomly distributed points, are studied in Reference [17,18]. Fractional differential equations with non-instantaneous impulses have recently been studied in Reference [19] but the meaning of the impulses are not random (as they are called in the paper) but arbitrary and the solutions are deterministic functions. Fractional equations with fixed, initially given non-instantaneous impulses, are studied over an interval with randomly varying trial lengths in Reference [20]. Fractional differential equations for random functions and with non-instantaneous impulses at fixed initially given intervals are studied in Reference [21]. Fractional differential equations with non-instantaneous impulses starting at randomly exponentially distributed times is studied in Reference [17].

In this paper, we study nonlinear fractional differential equations subject to non-instantaneous impulses starting abruptly at some random points. Note there are many types of fractional derivatives used and studied in the literature. Their application to differential equations leads to various types 
of initial conditions (for example, see References [4,22,23]. Due to the natural initial value problem in the application of Caputo fractional derivative and its real practical meaning we will study this type of fractional derivative. The study of impulsive moments in random time is very limited. Caputo fractional differential equations with instantaneously acting impulses at random times were studied in References [24-27] but there are some inaccuracies there in the mixing properties of deterministic variables and random variables and inaccuracies in the convergence of a sequence of real numbers to a random variable. Also, the type of the random distribution of the random variables is not taken into account. The model with any other types of fractional derivatives such as Riemann-Liouville fractional derivative or Atangana-Baleanu fractional derivative with random impulses are not studied yet. We study the case when the time between two consecutive moments of impulses is Erlang distributed random variable. In this paper the $p$-moment exponential stability of the solution is studied by employing Lyapunov functions. This study combines the ones of the theory of fractional differential equations and the probability theory. Note that similar problem is studied in Reference [17] for exponentially distributed random moments of impulses. Since the exponential distribution is a partial case of Erlang distribution, the results in this paper are a generalization of those in Reference [17]. Note also that in this paper the stability is studied without the restriction about the constant length of the randomly occurred intervals of impulses.

Note obtained sufficient conditions could be very useful for adequate modeling of real world phenomena with impulses occurring at random moments and better described by fractional differential equations (for several models with ordinary derivatives and random impulses see Section 3.3 in Reference [28]).

\section{Notes on Fractional Calculus}

In this paper we will consider fractional derivatives of order $q \in(0,1)$. The main types of fractional derivatives are:

- Caputo fractional derivative (see, for example, Section 1.4.1.3 Reference [22])

$$
{ }_{t_{0}}^{C} D^{q} m(t)=\frac{1}{\Gamma(1-q)} \int_{t_{0}}^{t}(t-s)^{-q} m^{\prime}(s) d s, \quad t \geq t_{0},
$$

where $\Gamma($.$) denotes the Gamma function.$

- Grunwald-Letnikov fractional derivative (see, for example, Section 1.4.1.2, Reference [22])

$$
{ }_{t_{0}}^{G L} D^{q} m(t)=\lim _{h \rightarrow 0} \frac{1}{h^{q}}\left[\frac{t-t_{0}}{h}\right]
$$

and the Grunwald-Letnikov fractional Dini derivative by

$$
{ }_{t_{0}}^{G L} D_{+}^{q} m(t)=\limsup _{h \rightarrow 0+} \frac{1}{h^{q}} \sum_{r=0}^{\left[\frac{t-t_{0}}{h}\right]}(-1)^{r}(q C r) m(t-r h), \quad t \geq t_{0}
$$

where $q C r=\frac{q(q-1) \ldots(q-r+1)}{r !}$ where $r \geq 0$ is an integer and $\left[\frac{t-t_{0}}{h}\right]$ denotes the integer part of the fraction $\frac{t-t_{0} r}{h}$.

According to Reference [23] the equality

$$
{ }_{t_{0}}^{C} D_{t}^{q} m(t)==_{t_{0}}^{G L} D_{t}^{q} m(t)-m\left(t_{0}\right) \frac{\left(t-t_{0}\right)^{-q}}{\Gamma(1-q)},
$$


holds for $t \in\left(t_{0}, b\right]$ where $m \in C^{1}\left[t_{0}, b\right]$.

Definition 1. ([29]) We say $m \in C^{q}\left(\left[t_{0}, T\right], \mathbb{R}^{n}\right)$ if $m(t)$ is differentiable (i.e., $m^{\prime}(t)$ exists), the Caputo derivative ${ }_{t_{0}}^{c} D^{q} m(t)$ exists and satisfies (1) for $t \in\left[t_{0}, T\right]$.

Remark 1. Definition 1 could be extended to any interval $I \subset \mathbb{R}_{+}$.

\section{Random Non-Instantaneous Impulses in Fractional Differential Equations}

We will start with non-instantaneous fractional differential equations in the case when the time of these impulses is determinic one and given initially. Let two increasing sequences of nonnegative points $\left\{T_{k}\right\}_{k=1}^{\infty}$ and $\left\{s_{i}\right\}_{i=1}^{\infty}$ be given such that $0<T_{i} \leq s_{i}<T_{i+1}, i=1,2, \ldots$ and $\lim _{k \rightarrow \infty} T_{k}=\infty$. Let $T_{0} \in\left[0, T_{1}\right) \cup \cup_{k=1}^{\infty}\left[s_{k}, T_{k+1}\right)$ be a given arbitrary point. Without loss of generality we will assume that $T_{0} \in\left[0, T_{1}\right)$ and denote $s_{0}=T_{0}$.

Consider the initial value problem (IVP) for the system of non-instantaneous impulsive fractional differential equations (NIFrDE) with fixed points of impulses

$$
\begin{aligned}
& { }_{T_{0}}^{c} D^{q} x=f(t, x(t)) \text { for } t \in\left(s_{k}, T_{k+1}\right], k=0,1,2, \ldots, \\
& x(t)=I_{i}\left(t, x\left(T_{i}-0\right)\right) \text { for } t \in\left(T_{i}, s_{i}\right], k=1,2, \ldots, \\
& x\left(T_{0}\right)=x_{0},
\end{aligned}
$$

where $x, x_{0} \in \mathbb{R}^{n}, f:[0, \infty) \times \mathbb{R}^{n} \rightarrow \mathbb{R}^{n}, f(t, 0)=0, \quad I_{i}:[0, \infty) \times \mathbb{R}^{n} \rightarrow \mathbb{R}^{n}, I_{i}(t, 0)=0$, $(i=1,2,3, \ldots)$.

In this paper, we will use Caputo fractional derivative with changeable lower limit at each point of jump. Note this approach is used for study various types of problems for deterministic non-instantaneous impulsive fractional differential equations in References [30-32].

Now we define fractional differential equations with random non-instantaneous impulses. Let $T_{0} \geq 0$ $\left\{d_{i}\right\}_{i=1}^{\infty}$ be given. Denote $d_{0}=0$.

Consider the following condition:

H1. The positive numbers $\left\{d_{k}\right\}_{k=1}^{\infty}$ are such that $\lim _{n \rightarrow \infty} \sum_{k=1}^{n} d_{k}=\infty$.

H2. The positive numbers $\left\{d_{k}\right\}_{k=1}^{\infty}$ are such that $\lim _{n \rightarrow \infty} \sum_{k=1}^{n} d_{k}=B<\infty$.

Let the probability space $(\Omega, \mathcal{F}, P)$ be given. Let a sequence of independent Erlang distributed random variables $\left\{\tau_{k}\right\}_{k=1}^{\infty}$ defined on the sample space $\Omega$.

Assume $\sum_{k=1}^{\infty} \tau_{k}=\infty$ with probability 1 .

We will use the following condition:

H3. The random variables $\left\{\tau_{k}\right\}_{k=1}^{\infty}, \tau_{k} \in \operatorname{Erlang}\left(\alpha_{k}, \lambda\right)$ are independent where the positive integer parameter $\alpha_{k}$ is the "shape" and the positive real parameter $\lambda$ is the "rate" with $\lim _{n \rightarrow \infty} \sum_{k=1}^{n} \alpha_{k}=\infty$.

We will give some properties of the Erlang distribution:

(P1) If $X \in \operatorname{Erlang}\left(\alpha_{1}, \lambda\right)$ and $Y \in \operatorname{Erlang}\left(\alpha_{2}, \lambda\right)$ are independent random variables, then $X+Y \in$ $\operatorname{Erlang}\left(\alpha_{1}+\alpha_{2}, \lambda\right)$

(P2) The cumulative distribution function (CDF) of $\operatorname{Erlang}(\alpha, \lambda)$ is

$$
F(x ; \alpha, \lambda)=1-e^{-\lambda x} \sum_{j=1}^{\alpha-1} \frac{(\lambda x)^{j}}{j !}, \quad x \geq 0 .
$$


Define the increasing sequence of random variables $\left\{\xi_{k}\right\}_{k=0}^{\infty}$ by

$$
\xi_{k}=T_{0}+\sum_{i=1}^{k} \tau_{i}+\sum_{i=1}^{k-1} d_{i}, \quad k=0,1,2, \ldots
$$

where $T_{0} \geq 0$ is a fixed point.

Also, $\Xi_{k}=\sum_{i=1}^{n} \tau_{i}=\xi_{k}-T_{0}-\sum_{i=1}^{k-1} d_{i}, k=1,2, \ldots$

From properties (P1) and (P2) of Erlang distribution it follows the result:

Proposition 1. Let condition (H3) be satisfied and $\Xi_{n}=\sum_{i=1}^{n} \tau_{i}, n$ is a natural number.

Then $\Xi_{n} \in \operatorname{Erlang}\left(\sum_{i=1}^{n} \alpha_{i}, \lambda\right)$, that is, CDF of $\Xi_{n}$ is

$$
F_{\Xi_{n}}(t)=P\left(\Xi_{n}<t\right)=1-e^{-\lambda t} \sum_{j=1}^{\sum_{i=1}^{n} \alpha_{i}-1} \frac{(\lambda t)^{j}}{j !}, \quad t \geq 0 .
$$

The meaning of the above defined random variables and deterministic sequence is:

- $\quad$ the waiting time of the $k$-th impulse after the stop of action of $(k-1)$-st impulse is measured by random variable $\tau_{k}$;

- the length of the time interval of acting the $k$-th impulse is given by $d_{k}$;

- the length of time until $k$ impulses occur for $t \geq T_{0}$ is measured by the random variable $\xi_{k}$.

Let for any $k=1,2, \ldots$ the point $t_{k}$ be an arbitrary value of the random variable $\tau_{k}$. Define two increasing sequences of points $\left\{T_{k}\right\}_{k=1}^{\infty}$ and $\left\{s_{k}\right\}_{k=1}^{\infty}$ by

$$
T_{k}=T_{0}+\sum_{i=1}^{k} t_{i}+\sum_{i=1}^{k-1} d_{i}
$$

and

$$
s_{0}=T_{0}, \quad s_{k}=T_{k}+d_{k}=T_{0}+\sum_{i=1}^{k} t_{i}+\sum_{i=1}^{k} d_{i}, \quad k=1,2,3 \ldots
$$

Also, $T_{k+1}-s_{k}=t_{k+1}$, that is, it is a value of the random variable $\tau_{k+1}$.

Note $T_{k}$ and $s_{k}$ are values of the random variables $\xi_{k}$ and $\xi_{k}+d_{k}, k=1,2, \ldots$, respectively and $s_{k-1} \leq T_{k} \leq s_{k}, k=1,2, \ldots$

Consider the IVP for NIFrDE (2). The solution of (2) depends not only on the initial condition $\left(T_{0}, x_{0}\right)$ but on the moments of impulses $T_{k}, k=1,2, \ldots$, that is, the solution depends on the chosen arbitrary values $t_{k}$ of the random variables $\tau_{k}, k=1,2, \ldots$ We denote the solution of (2) by $x\left(t ; T_{0}, x_{0},\left\{T_{k}\right\}\right)$. Assume $x\left(T_{k} ; T_{0}, x_{0},\left\{T_{k}\right\}\right)=\lim _{t \rightarrow T_{k}-0} x\left(t ; T_{0}, x_{0},\left\{T_{k}\right\}\right)$ for any $k=1,2, \ldots$ The set of all solutions $x\left(t ; T_{0}, x_{0},\left\{T_{k}\right\}\right)$ of (2) generates a stochastic process with state space $\mathbb{R}^{n}$.

Consider the IVP for fractional differential equations with random moments of non-instantaneous impulses (RNIFrDE)

$$
\begin{aligned}
& { }_{T_{0}}^{c} D^{q} x(t)=f(t, x(t)) \text { for } t \geq T_{0}, \xi_{k}+d_{k}<t<\xi_{k+1}, k=0,1, \ldots, \\
& x(t)=I_{k}\left(t, x\left(\xi_{k}\right)\right) \text { for } \xi_{k}<t<\xi_{k}+d_{k}, k=1,2, \ldots, \\
& x\left(T_{0}\right)=x_{0},
\end{aligned}
$$

where $x_{0} \in \mathbb{R}^{n}, d_{0}=0, f:[0, \infty) \times \mathbb{R}^{n} \rightarrow \mathbb{R}^{n}, I_{k}:[0, \infty) \times \mathbb{R}^{n} \rightarrow \mathbb{R}^{n},(k=1,2,3, \ldots)$.

Definition 2. [33]. For any given values $t_{k}$ of the random variables $\tau_{k}, k=1,2,3, \ldots$ respectively, the solution $x\left(t ; T_{0}, x_{0},\left\{T_{k}\right\}\right)$ of the corresponding IVP for the NIFrDE (2) is called a sample path solution of the IVP for the RNIFrDE (7). 
Definition 3. [33]. The stochastic process $x\left(t ; T_{0}, x_{0},\left\{\tau_{k}\right\}\right)$ is said to be a solution of the IVP for RNIFrDE (7) if for any values $t_{k}$ of the random variables $\tau_{k}, k=1,2, \ldots$ the corresponding function $x\left(t ; T_{0}, x_{0},\left\{T_{k}\right\}\right)$ is a sample path solution of the IVP for RNIFrDE (7).

Remark 2. Note that the Caputo fractional derivative in RNIFrDE (7) has a changeable lower limit on each interval without impulses. This lower limit is a value of the random variable $\xi_{k}, k=1,2, \ldots$

Any sample path solution $x\left(t ; T_{0}, x_{0},\left\{T_{k}\right\}\right) \in C^{q}\left(\left(s_{k}, T_{k+1}\right], \mathbb{R}^{n}\right), k=0,1,2, \ldots$

Definition 4. We will say that the stochastic processes $y(t)$ and $u(t)$ satisfy the inequality $y(t) \leq u(t)$ for $t \in J \subset \mathbb{R}$ if the state space of the stochastic processes $v(t)=y(t)-v(t)$ is $(-\infty, 0]$.

\section{Preliminary Results for Erlang Distributed Moments of Impulses}

We will give some results about Erlnag distributed moments of impulses studied in Reference [18] and applied to study ordinary differential equation with random non-instantaneous impulses.

For any $t \geq T_{0}$ consider the events

$$
\begin{gathered}
S_{0}(t)=\left\{\omega \in \Omega: 0<t-T_{0}<\tau_{1}(\omega)\right\}, \\
S_{k}(t)=\left\{\omega \in \Omega: \xi_{k}(\omega)+d_{k}<t<\xi_{k+1}(\omega)\right\}, \quad k=1,2, \ldots,
\end{gathered}
$$

and

$$
W_{k}(t)=\left\{\omega \in \Omega: \xi_{k}(\omega)<t<\xi_{k}(\omega)+d_{k}\right\}, \quad k=1,2, \ldots,
$$

where the random variables $\xi_{k}, k=1,2, \ldots$ are defined by (4).

Lemma 1. [18]. For any $t \geq T_{0}$ the inequality

$$
P\left(S_{0}(t)\right) \leq e^{-\lambda\left(t-T_{0}\right)} \sum_{i=1}^{\alpha_{1}-1} \frac{\left(\lambda\left(t-T_{0}\right)\right)^{i}}{(i) !}
$$

holds.

Lemma 2. ( Upper bound of $S_{k}(t)$ ) [18]. Let condition (H3) and one of (H1) or (H2) be satisfied. Then for any natural number $j$ we have

$$
\begin{aligned}
P\left(S_{j}(t)\right) & \leq e^{-\lambda\left(t-T_{0}-\sum_{i=1}^{j} d_{i}\right)} \sum_{i=\sum_{m=1}^{j} \alpha_{m}}^{\sum_{m=1}^{j+1} \alpha_{m}-1} \frac{\left(\lambda\left(t-T_{0}-\sum_{i=1}^{j} d_{i}\right)\right)^{i}}{i !} \\
& \leq e^{-\lambda\left(t-T_{0}-\sum_{i=1}^{j} d_{i}\right)} \sum_{i=1}^{\sum_{m=1}^{j+1} \alpha_{m}-1} \frac{\left(\lambda\left(t-T_{0}-\sum_{i=1}^{j} d_{i}\right)\right)^{i}}{i !}
\end{aligned}
$$

holds.

Lemma 3. (Upper bound of $W_{k}(t)$ [18]. Let condition (H3) and one of (H1) or (H2) be satisfied. Then for any natural number $j$ we have

$$
\begin{aligned}
& P\left(W_{j}(t)\right) \\
& \leq e^{-\lambda\left(t-T_{0}-\sum_{i=1}^{j} d_{i}\right)} \sum_{i=1}^{\sum_{m=1}^{j} \alpha_{m}-1} \frac{\left(\lambda\left(t-T_{0}-\sum_{i=1}^{j} d_{i}\right)\right)^{i}-\left(\lambda\left(t-T_{0}-\sum_{i=1}^{j-1} d_{i}\right)\right)^{i} e^{-\lambda d_{k}}}{i !}
\end{aligned}
$$

for $j \leq k$. 


\section{Linear Fractional Differential Equation with Random Impulses}

Consider the scalar linear fractional differential equation with non-instantaneous random moments of impulses:

$$
\begin{aligned}
& { }_{T_{0}}^{c} D^{q} u=-a_{k} u \text { for } \xi_{k}+d_{k}<t<\xi_{k+1}, k=0,1,2, \ldots, \\
& u(t)=b_{k} u\left(\xi_{k}\right), \text { for } \xi_{k}<t<\xi_{k}+d_{k}, k=1,2, \ldots, \\
& u\left(T_{0}\right)=u_{0},
\end{aligned}
$$

where $u_{0} \in \mathbb{R}, a_{k} \geq 0, k=0,1,2, \ldots, b_{k} \neq 1,(k=, 2, \ldots)$ and $d_{k}>0$ are given real constants, $d_{0}=0$.

\section{Lemma 4. Let}

1. Condition (H3) and one of (H1) and (H2) be satisfied.

2. Let there exist positive constants $M_{k}, \mu_{k}, k=0,1,2 \ldots$ such that

$$
E_{q}\left(-a_{0}\left(t-T_{0}\right)^{q}\right) \sum_{i=1}^{\alpha_{1}-1} \frac{\left(\lambda\left(t-T_{0}\right)\right)^{i}}{(i) !} \leq M_{0} e^{\mu_{0}\left(t-T_{0}\right)}, \quad t \geq T_{0}
$$

and

$$
\frac{B\left(\lambda\left(t-T_{0}\right)\right)^{\alpha_{k+1}}+1}{\lambda\left(t-T_{0}-\sum_{i=1}^{k} d_{i}\right) \Gamma\left(\sum_{m=1}^{k} \alpha_{m}\right)} \prod_{i=1}^{k}\left|b_{i} e^{\lambda d_{i}}\left(\lambda\left(t-T_{0}\right)\right)^{\alpha_{i}}\right| \leq M_{k} e^{\mu_{k}\left(t-T_{0}\right)}, \quad t \geq T_{0}
$$

with

$$
\sum_{k=0}^{\infty} M_{k} e^{\mu_{k}\left(t-T_{0}\right)} \leq M e^{\mu\left(t-T_{0}\right)}
$$

where $M, \mu>0: \mu<\lambda$.

Then the solution $u\left(t ; T_{0}, x_{0},\left\{\tau_{k}\right\}\right)$ of the IVP for the linear RNIFrDE (10) is given by the formula

$$
u\left(t ; T_{0}, u_{0},\left\{\tau_{k}\right\}\right)=\left\{\begin{array}{c}
u_{0} \prod_{i=1}^{k} b_{i}\left(\prod_{i=0}^{k-1} E_{q}\left(-a_{i}\left(\tau_{i+1}\right)^{q}\right)\right) \\
\text { for } \xi_{k}<t \leq \xi_{k}+d_{k}, k=1,2, \ldots, \\
u_{0} \prod_{i=1}^{k} b_{i}\left(\prod_{i=0}^{k-1} E_{q}\left(-a_{i}\left(\tau_{i+1}\right)^{q}\right)\right) E_{q}\left(-a_{k}\left(t-\xi_{k}-d_{k}\right)^{q}\right) \\
\text { for } \xi_{k}+d_{k}<t \leq \xi_{k+1}, k=0,1,2, \ldots,
\end{array}\right.
$$

where $E_{q}$ is the Mittag-Leffler function (with one parameter) and the expected value of the solution satisfies the inequality

$$
E\left(\left|u\left(t ; T_{0}, u_{0},\left\{\tau_{k}\right\}\right)\right|\right) \leq\left|u_{0}\right| e^{-(\lambda-\mu)\left(t-T_{0}\right)}, \quad t \geq T_{0} .
$$

Proof. The sample path solution of (10) is given by (note the product is 1 if $k=1$ )

$$
u\left(t ; T_{0}, x_{0},\left\{T_{k}\right\}\right)=\left\{\begin{array}{c}
u_{0} \prod_{i=1}^{k} b_{i}\left(\prod_{i=0}^{k-1} E_{q}\left(-a_{i}\left(T_{i+1}-T_{i}-d_{i}\right)^{q}\right)\right) \\
\text { for } t \in\left(T_{k}, T_{k}+d_{k}\right], k=1,2, \ldots, \\
u_{0} \prod_{i=1}^{k} b_{i}\left(\prod_{i=0}^{k-1} E_{q}\left(-a_{i}\left(T_{i+1}-T_{i}-d_{i}\right)^{q}\right)\right) E_{q}\left(-a_{k}\left(t-T_{k}-d_{k}\right)^{q}\right) \\
\text { for } t \in\left(T_{k}+d_{k}, T_{k+1}\right], k=0,1,2, \ldots
\end{array}\right.
$$

The equality (14) and Definition 3 establish (12) for the IVP for the linear RNIFrDE (10). 
Then the expected value of the solution of the IVP for the scalar linear RNIFrDE (10) satisfies

$$
\begin{aligned}
E\left(\left|u\left(t ; T_{0}, u_{0},\left\{\tau_{k}\right\}\right)\right|\right)= & E\left(\left|u\left(t ; T_{0}, u_{0},\left\{\tau_{k}\right\}\right)\right| \mid S_{0}(t)\right) P\left(S_{0}(t)\right) \\
& +\sum_{k=1}^{\infty} E\left(\left|u\left(t ; T_{0}, u_{0},\left\{\tau_{k}\right\}\right)\right| \mid S_{k}(t)\right) P\left(S_{k}(t)\right) \\
& +\sum_{k=1}^{\infty} E\left(\left|u\left(t ; T_{0}, u_{0},\left\{\tau_{k}\right\}\right)\right| \mid W_{k}(t)\right) P\left(W_{k}(t)\right) .
\end{aligned}
$$

From Equation (12), the independence of the random variables $\tau_{k}, k=1,2, \ldots$, the inequalities $0<E_{q}(-A) \leq 1$ for $A \geq 0, E(\eta) \leq E(\xi)$ for the random variables $\eta, \xi: 0 \leq \eta \leq \xi$ we see

- $\quad$ for $\xi_{0}<t \leq \xi_{1}$

$$
\begin{aligned}
& E\left(\left|u\left(t ; T_{0}, u_{0},\left\{\tau_{k}\right\}\right)\right| \mid S_{0}(t)\right)=E\left(\left|u_{0}\right| E_{q}\left(-a_{0}\left(t-T_{0}\right)^{q}\right)\right) \\
& =\left|u_{0}\right| E_{q}\left(-a_{0}\left(t-T_{0}\right)^{q}\right) \leq\left|u_{0}\right| ;
\end{aligned}
$$

- $\quad$ for $\xi_{k}+d_{k}<t \leq \xi_{k+1}, k$ is a natural number we get $t-\xi_{k}-d_{k}<0, E_{q}\left(-a_{k}\left(t-\xi_{k}-d_{k}\right)^{q}\right) \leq 1$ and

$$
\begin{aligned}
& E\left(\left|u\left(t ; T_{0}, u_{0},\left\{\tau_{k}\right\}\right)\right| \mid S_{k}(t)\right) \\
& =E\left(\left|u_{0}\right| \prod_{i=1}^{k}\left|b_{i}\right|\left(\prod_{i=0}^{k-1} E_{q}\left(-a_{i}\left(\tau_{i+1}\right)^{q}\right)\right) E_{q}\left(-a_{k}\left(t-\xi_{k}-d_{k}\right)^{q}\right)\right) \\
& \leq\left|u_{0}\right| \prod_{i=1}^{k}\left|b_{i}\right| \prod_{i=0}^{k-1} E\left(E_{q}\left(-a_{i}\left(\tau_{i+1}\right)^{q}\right)\right) \leq\left|u_{0}\right| \prod_{i=1}^{k}\left|b_{i}\right|
\end{aligned}
$$

- $\quad$ for $\xi_{k}<t \leq \xi_{k}+d_{k}, k$ is a natural number, we get $E_{q}\left(-a_{i}\left(\tau_{i+1}\right)^{q}\right) \leq 1$ and

$$
\begin{aligned}
& E\left(\left|u\left(t ; T_{0}, u_{0},\left\{\tau_{k}\right\}\right)\right| \mid W_{k}(t)\right)=\left|u_{0}\right| \prod_{i=1}^{k}\left|b_{i}\right|\left(E \prod_{i=0}^{k-1} E_{q}\left(-a_{i}\left(\tau_{i+1}\right)^{q}\right)\right) \\
& \leq\left|u_{0}\right| \prod_{i=1}^{k}\left|b_{i}\right| .
\end{aligned}
$$

Apply inequalities (15)-(17), Lemmas 1-3 to inequality (14) and obtain for the expected value

$$
\begin{aligned}
& E\left(\left|u\left(t ; T_{0}, u_{0},\left\{\tau_{k}\right\}\right)\right|\right) \\
& \leq\left|u_{0}\right| E_{q}\left(-a_{0}\left(t-T_{0}\right)^{q}\right) P\left(S_{0}(t)\right)+\left|u_{0}\right| \sum_{k=1}^{\infty} \prod_{i=1}^{k}\left|b_{i}\right|\left(P\left(S_{k}(t)\right)+P\left(W_{k}(t)\right)\right) \\
& \leq\left|u_{0}\right| e^{-\lambda\left(t-T_{0}\right)}\left\{E_{q}\left(-a_{0}\left(t-T_{0}\right)^{q}\right) \sum_{i=1}^{\alpha_{1}-1} \frac{\left(\lambda\left(t-T_{0}\right)\right)^{i}}{(i) !}\right. \\
& +\sum_{k=1}^{\infty} \prod_{i=1}^{k}\left|b_{i}\right|\left[e ^ { \lambda \sum _ { i = 1 } ^ { k } d _ { i } } \left(\sum_{i=1}^{\sum_{m=1}^{k+1} \alpha_{m}-1} \frac{\left(\lambda\left(t-T_{0}-\sum_{i=1}^{k} d_{i}\right)\right)^{i}}{i !}\right.\right. \\
& \left.\left.\left.+\sum_{i=1}^{\sum_{m=1}^{\alpha} \alpha_{m}-1} \frac{\left(\lambda\left(t-T_{0}-\sum_{i=1}^{k} d_{i}\right)\right)^{i}-\left(\lambda\left(t-T_{0}-\sum_{i=1}^{k-1} d_{i}\right)\right)^{i} e^{\lambda d_{k}}}{i !}\right)\right]\right\} .
\end{aligned}
$$

Now apply the inequalities $x^{a-1} e^{-x}<\Gamma(a, x)<B x^{a-1} e^{-x}$ (see Equation (1.5) and (2.3) [1]) for $a>1, B>1, x>\frac{B}{B-1}(a-1)$, 


$$
\sum_{i=1}^{b} \frac{c^{i}}{i !}=e^{c} \frac{\Gamma(1+b, c)}{\Gamma(1+b)}-1<\frac{B c^{b}}{\Gamma(1+b)}-1, \quad \Gamma(a, x) \leq \frac{x^{a} e^{-x}}{x-(a-1)}, a \geq 1, x>0,
$$

for any $t>T_{0}+\sum_{i=1}^{k} d_{i}$ we get $B x-x>B(a-1), B(x-a+1)>x$ or $B(c-b)>c$ or $\lambda\left(t-T_{0}-\right.$ $\left.\sum_{i=1}^{k} d_{i}\right)>\sum_{m=1}^{k+1} \alpha_{m}-1$ and $B>\frac{\lambda\left(t-T_{0}-\sum_{i=1}^{k} d_{i}\right)}{\lambda\left(t-T_{0}-\sum_{i=1}^{k} d_{i}\right)-\sum_{m=1}^{k+1} \alpha_{m}+1}>1$

$$
\begin{gathered}
B>\frac{\lambda\left(t-T_{0}-\sum_{i=1}^{k} d_{i}\right)}{\lambda\left(t-T_{0}-\sum_{i=1}^{k} d_{i}\right)-\sum_{m=1}^{k+1} \alpha_{m}} \geq \frac{\lambda\left(t-T_{0}-\sum_{i=1}^{k} d_{i}\right)}{\lambda\left(t-T_{0}-\sum_{i=1}^{k} d_{i}\right)-\sum_{m=1}^{k+1} \alpha_{m}+1}>1, \\
\sum_{m=1}^{\sum_{i=1}^{k+1} \alpha_{m}-1} \frac{\left(\lambda\left(t-T_{0}-\sum_{i=1}^{k} d_{i}\right)\right)^{i}}{i !} \\
=e^{\left(\lambda\left(t-T_{0}-\sum_{i=1}^{k} d_{i}\right)\right)} \frac{\Gamma\left(\sum_{m=1}^{k+1} \alpha_{m}, \lambda\left(t-T_{0}-\sum_{i=1}^{k} d_{i}\right)\right.}{\Gamma\left(\sum_{m=1}^{k+1} \alpha_{m}\right)}-1 \\
\leq \frac{B\left(\lambda\left(t-T_{0}-\sum_{i=1}^{k} d_{i}\right)\right)_{m=1}^{\sum_{m}^{k+1} \alpha_{m}-1}}{\Gamma\left(\sum_{m=1}^{k+1} \alpha_{m}\right)}-1
\end{gathered}
$$

and

$$
\begin{aligned}
& \sum_{i=1}^{k} \frac{\sum_{m=1}^{\alpha_{m}-1}}{\left(\lambda\left(t-T_{0}-\sum_{i=1}^{k} d_{i}\right)\right)^{i}-\left(\lambda\left(t-T_{0}-\sum_{i=1}^{k-1} d_{i}\right)\right)^{i} e^{\lambda d_{k}}} \\
& i ! \\
& <B \frac{\left(\lambda\left(t-T_{0}-\sum_{i=1}^{k} d_{i}\right)\right)^{\sum_{m=1}^{k} \alpha_{m}-1}}{\Gamma\left(\sum_{m=1}^{k} \alpha_{m}\right)}-e^{\lambda d_{k}} \frac{\left(\lambda\left(t-T_{0}-\sum_{i=1}^{k-1} d_{i}\right)\right)^{\sum_{m=1}^{k} \alpha_{m}}}{\Gamma\left(\sum_{m=1}^{k} \alpha_{m}\right)} .
\end{aligned}
$$
we get

Then using that the function $\Gamma(u)$ is increasing for $u \geq 2$, that is, $\Gamma\left(\sum_{m=1}^{k+1} \alpha_{m}\right) \geq \Gamma\left(\sum_{m=1}^{k} \alpha_{m}\right)$

$$
\begin{aligned}
& E\left(\left|u\left(t ; T_{0}, u_{0},\left\{\tau_{k}\right\}\right)\right|\right) \\
& \leq\left|u_{0}\right| e^{-\lambda\left(t-T_{0}\right)}\left\{E_{q}\left(-a_{0}\left(t-T_{0}\right)^{q}\right) \sum_{i=1}^{\alpha_{1}-1} \frac{\left(\lambda\left(t-T_{0}\right)\right)^{i}}{(i) !}\right. \\
& +\sum_{k=1}^{\infty} \prod_{i=1}^{k}\left|b_{i} e^{\lambda d_{i}}\right|\left[\frac{B\left(\lambda\left(t-T_{0}-\sum_{i=1}^{k} d_{i}\right)\right)^{\sum_{m=1}^{k+1} \alpha_{m}-1}}{\Gamma\left(\sum_{m=1}^{k+1} \alpha_{m}\right)}-1\right. \\
& \left.\left.+B \frac{\left(\lambda\left(t-T_{0}-\sum_{i=1}^{k} d_{i}\right)\right)^{k} \sum_{m=1}^{k} \alpha_{m}-1}{\Gamma\left(\sum_{m=1}^{k} \alpha_{m}\right)}-e^{\lambda d_{k}} \frac{\left(\lambda\left(t-T_{0}-\sum_{i=1}^{k-1} d_{i}\right)\right)^{\sum_{m=1}^{k} \alpha_{m}}}{\Gamma\left(\sum_{m=1}^{k} \alpha_{m}\right)}\right]\right\} \\
& \leq\left|u_{0}\right| e^{-\lambda\left(t-T_{0}\right)}\left\{E_{q}\left(-a_{0}\left(t-T_{0}\right)^{q}\right) \sum_{i=1}^{\alpha_{1}-1} \frac{\left(\lambda\left(t-T_{0}\right)\right)^{i}}{(i) !}\right. \\
& \left.+\sum_{k=1}^{\infty} \prod_{i=1}^{k}\left|b_{i} e^{\lambda d_{i}}\right|\left[\frac{B\left(\left(\lambda\left(t-T_{0}-\sum_{i=1}^{k} d_{i}\right)\right)^{\alpha_{k+1}}+1\right)\left(\lambda\left(t-T_{0}-\sum_{i=1}^{k} d_{i}\right)\right)^{\sum_{m=1}^{k} \alpha_{m}}}{\lambda\left(t-T_{0}-\sum_{i=1}^{k} d_{i}\right) \Gamma\left(\sum_{m=1}^{k} \alpha_{m}\right)}\right]\right\} \\
& \leq\left|u_{0}\right| e^{-\lambda\left(t-T_{0}\right)}\left\{E_{q}\left(-a_{0}\left(t-T_{0}\right)^{q}\right) \sum_{i=1}^{\alpha_{1}-1} \frac{\left(\lambda\left(t-T_{0}\right)\right)^{i}}{(i) !}\right. \\
& +\sum_{k=1}^{\infty} \prod_{i=1}^{k}\left|b_{i} e^{\lambda d_{i}}\left(\lambda\left(t-T_{0}-\sum_{j=1}^{k} d_{j}\right)\right)^{\alpha_{i}}\right| \frac{B\left(\left(\lambda\left(t-T_{0}-\sum_{i=1}^{k} d_{i}\right)\right)^{\alpha_{k+1}}+1\right)}{\lambda\left(t-T_{0}-\sum_{i=1}^{k} d_{i}\right) \Gamma\left(\sum_{m=1}^{k} \alpha_{m}\right)} \\
& \leq\left|u_{0}\right| e^{-\lambda\left(t-T_{0}\right)}\left\{E_{q}\left(-a_{0}\left(t-T_{0}\right)^{q}\right) \sum_{i=1}^{\alpha_{1}-1} \frac{\left(\lambda\left(t-T_{0}\right)\right)^{i}}{(i) !}\right. \\
& +\sum_{k=1}^{\infty} \frac{B\left(\lambda\left(t-T_{0}\right)\right)^{\alpha_{k+1}}+1}{\lambda\left(t-T_{0}-\sum_{i=1}^{k} d_{i}\right) \Gamma\left(\sum_{m=1}^{k} \alpha_{m}\right)} \prod_{i=1}^{k}\left|b_{i} e^{\lambda d_{i}}\left(\lambda\left(t-T_{0}\right)\right)^{\alpha_{i}}\right| .
\end{aligned}
$$


Then according to condition 2 we get

$$
E\left(\left|u\left(t ; T_{0}, u_{0},\left\{\tau_{k}\right\}\right)\right|\right) \leq\left|u_{0}\right| e^{-(\lambda-\mu)\left(t-T_{0}\right)}, t \geq T_{0} .
$$

\section{Lyapunov Functions}

Definition 5. Let $J \subset \mathbb{R}_{+}$and $\Delta \subset \mathbb{R}^{n}, 0 \in \Delta$. The function $V(t, x): J \times \Delta \rightarrow \mathbb{R}_{+}, V(t, 0) \equiv 0$ is from the class $\Lambda(J, \Delta)$ if it is continuous on $J \times \Delta$ and locally Lipschitzian with respect to its second argument.

We will use fractional Dini derivatives of Lyapunov function $V \in \Lambda\left(\left[T_{0}, \infty\right), \Delta\right)$ defined by:

$$
\begin{aligned}
& { }^{c} D_{+}^{q} V\left(t, x ; T_{0}\right)=\limsup _{h \rightarrow 0^{+}} \frac{1}{h^{q}}\left\{V(t, x)-\left[\frac{t-T_{0}}{h}\right]\right. \\
& \quad \text { for } t \geq T_{0},
\end{aligned}
$$

where $q C r=\frac{q(q-1)(q-2) \ldots(q-r+1)}{r !}$.

\section{7. $p$-Moment Exponential Stability for RNIFrDE}

Definition 6. Let $p>0$. Then the trivial solution of the RNIFrDE (7) is

- $\quad$-moment exponentially stable if for any initial point $\left(T_{0}, x_{0}\right) \in \mathbb{R}_{+} \times \mathbb{R}^{n}$ there exist constants $\alpha, \mu>0$ such that $\left.E\left[\| x\left(t ; T_{0}, x_{0},\left\{\tau_{k}\right)\right\}\right) \|^{p}\right]<\alpha\left\|x_{0}\right\|^{p} e^{-\mu\left(t-T_{0}\right)}$ for all $t>T_{0}$, where $x\left(t ; T_{0}, x_{0},\left\{\tau_{k}\right)\right\}$ is the solution of the IVP for the RNIFrDE (7);

- eventually p-moment exponentially stable if for any initial point $\left(T_{0}, x_{0}\right) \in \mathbb{R}_{+} \times \mathbb{R}^{n}$ there exist constants $\Theta=\Theta\left(T_{0}\right), \alpha, \mu>0$ such that $\left.E\left[\| x\left(t ; T_{0}, x_{0},\left\{\tau_{k}\right)\right\}\right) \|^{p}\right]<\alpha\left\|x_{0}\right\|^{p} e^{-\mu\left(t-T_{0}\right)}$ for all $t>T_{0}+\Theta$.

Theorem 1. Let the following conditions be satisfied:

1. Conditions (H3) and one of (H1) and (H2) hold.

2. The function $V \in \Lambda\left([0, \infty), \mathbb{R}^{n}\right)$, for any $x \in \mathbb{R}^{n}$ the inequality $V(t, x)$ is nondecreasing in $t \geq 0$ and there exist positive constants $a, b$ such that

(i) $a\|x\|^{p} \leq V(t, x) \leq b\|x\|^{p}$ for $t \geq T_{0} x \in \mathbb{R}^{n}$;

(ii) there exists a constant $m \geq 0$ such that for any $T \in \mathbb{R}_{+}, x_{0} \in \mathbb{R}^{n}$ the inequality

$$
{ }^{c} D_{+}^{q} V(t, x ; T) \leq-m V(t, x), \text { for } t \geq T, x \in \mathbb{R}^{n}
$$

holds;

(iii) for any $k=1,2, \ldots$ there exist functions $w_{k} \in C\left(\mathbb{R}_{+}, \mathbb{R}_{+}\right)$and positive constants $C$ : $C<e^{-\lambda d}$ and $C_{k}: w_{k}(t) \leq C_{k} \leq C<1$ for $t \geq 0$ such that

$$
V\left(t, I_{k}(t, x)\right) \leq w_{k}(t) V(t, x) \quad \text { for } t \geq 0, x \in \mathbb{R}^{n} .
$$

3. Let condition 2 of Lemma 4 is satisfied with $b_{k}=C_{k}$ and $a_{k}=m$.

Then the trivial solution of the RNIFrDE (7) is p-moment exponentially stable.

Proof. Choose an arbitrary initial data $\left(T_{0}, x_{0}\right) \in \mathbb{R}_{+} \times \mathbb{R}^{n}$ and let the stochastic process $x_{\tau}(t)=$ $x\left(t ; T_{0}, x_{0},\left\{\tau_{k}\right)\right\}$ be the solution of IVP for RNIFrDE (7). 
Now consider the IVP for the scalar linear RNIFrDE

$$
\begin{aligned}
& \stackrel{c}{T}_{T_{0}} D_{+}^{q} u(t)=-m u(t) \text { for } \xi_{k}+d<t<\xi_{k+1}, k=0,1,2, \ldots, \\
& u(t)=C_{k} u\left(\xi_{k}\right) \text { for } \xi_{k}<t<\xi_{k}+d, k=1,2, \ldots, \\
& u\left(T_{0}\right)=V\left(T_{0}, x_{0}\right) .
\end{aligned}
$$

According to Lemma 4 the IVP for RNIFrDE (23) has a solution $u\left(t ; T_{0}, V\left(T_{0}, x_{0}\right),\left\{\tau_{k}\right\}\right)$ given by (12) with $a=m, b_{k}=C_{k}$ and $u_{0}=V\left(T_{0}, x_{0}\right)$ and inequality (13) holds.

Choose arbitrary values $t_{k}$ of the random variables $\tau_{k}, k=1,2, \ldots$ Define $T_{k}=T_{0}+\sum_{i=1}^{k} t_{i}+$ $k d, k=1,2, \ldots$ and $s_{k}=T_{k}+d$. Note $T_{k}$ are values of the random variables $\xi_{k}$ and $s_{k}$ are values of random variables $\xi_{k}+d$. The corresponding function $x\left(t ; T_{0}, x_{0},\left\{T_{k}\right\}\right)$ is a sample path solution of the IVP for RNIFrDE (7) and the corresponding function $u(t)=u\left(t ; T_{0}, V\left(T_{0}, x_{0}\right),\left\{T_{k}\right\}\right)$ is a sample path solution of the IVP for RNIFrDE (23).

Define $v(t)=V\left(t, x\left(t ; T_{0}, x_{0},\left\{T_{k}\right\}\right)\right)$ for $t \geq T_{0}, t \neq T_{k}$ and $v\left(T_{k}\right)=V\left(T_{k}, x\left(T_{k}-0 ; T_{0}, x_{0},\left\{T_{k}\right\}\right)\right)$.

Let $t \in\left(T_{k}, s_{k}\right], k=0,1,2, \ldots$. From the continuity and monotonicity of the function $V(t, x)$ and condition 2(iii) we obtain

$$
\begin{aligned}
v(t) & =V\left(t, x\left(t ; T_{0}, x_{0},\left\{T_{k}\right\}\right)\right)=V\left(t, I_{k}\left(t, x\left(T_{k}-0 ; T_{0}, x_{0},\left\{T_{k}\right\}\right)\right)\right) \\
& \leq w_{k}(t) V\left(t, x\left(T_{k}-0 ; T_{0}, x_{0},\left\{T_{k}\right\}\right)\right) \leq w_{k}(t) V\left(T_{k}, x\left(T_{k}-0 ; T_{0}, x_{0},\left\{T_{k}\right\}\right)\right) \\
& =w_{k}(t) v\left(T_{k}\right) \leq C_{k} v\left(T_{k}\right), \quad t \in\left(s_{k}, T_{k}\right], k=0,1,2, \ldots
\end{aligned}
$$

Now, consider any interval $\left(s_{k}, T_{k+1}\right]$. Then using $v\left(s_{k}\right)=V\left(s_{k}, x\left(s_{k} ; T_{0}, x_{0},\left\{T_{k}\right\}\right)\right)$ with $x_{k}=x\left(s_{k}\right)$ and $x(t)=x\left(t ; T_{0}, x_{0},\left\{T_{k}\right\}\right)$ we obtain

$$
\begin{aligned}
& v(t)-v\left(s_{k}\right)-\sum_{r=1}^{\left[\frac{t-s_{k}}{h}\right]}(-1)^{r+1} q C r\left[v(t-r h)-v\left(s_{k}\right)\right] \\
& =\left\{V(t, x(t))-V\left(s_{k}, x_{k}\right)\right. \\
& \quad\left[\frac{t-s_{k}}{h}\right] \\
& -\sum_{r=1}^{h}(-1)^{r+1} q C r\left[V\left(t-r h, x(t)-h^{q} f(t, x(t))-V\left(s_{k}, x_{k}\right)\right]\right\} \\
& \quad\left[\frac{t-s_{k}}{\sum_{r=1}^{h}}\right]
\end{aligned}
$$

Since $x(t) \in C^{q}\left(\left(T_{k}, T_{k+1}\right], \mathbb{R}^{n}\right)$ from Remark 2 we have ${ }_{s_{k}}^{c} D^{q} x(t)=_{s_{k}}^{c} D_{+}^{q} x(t)$ and

$$
\begin{aligned}
c_{s_{k}}^{c} D_{+}^{q} x(t) & =\limsup _{h \rightarrow 0+} \frac{1}{h^{q}}\left[x(t)-x\left(s_{k}\right)-\left[\frac{t-s_{k}}{h}\right]\right. \\
& =f(t, x(t))
\end{aligned}
$$

or

$$
x(t)-h^{q} f(t, x(t))=S_{k}(x(t), h)+x\left(s_{k}\right)+\Lambda\left(h^{q}\right)
$$


with $\frac{\Lambda\left(h^{q}\right)}{h^{q}} \rightarrow 0$ as $h \rightarrow 0$ where

$$
S_{k}(x(t), h)=\sum_{r=1}^{\left[\frac{t-s_{k}}{h}\right]}(-1)^{r+1} q C r\left[x(t-r h)-x_{k}\right] .
$$

Therefore, we get

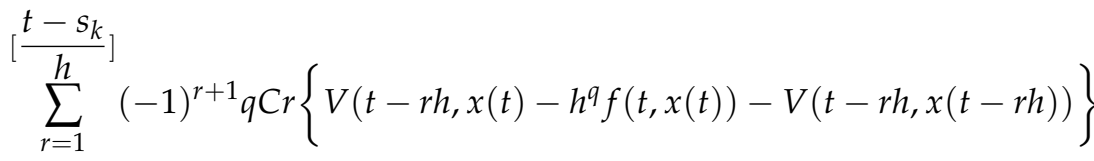

$$
\begin{aligned}
& \leq L\left\|\sum_{r=1}^{\left[\frac{t-s_{k}}{h}\right]} q C r\left(S_{k}(x(t), h) k+\Lambda\left(h^{q}\right)-\left(x(t-r h)-x_{k}\right)\right)\right\| \\
& \leq L \| \sum_{r=1}^{\left[\frac{t-s_{k}}{h}\right]}(-1)^{r+1} q C r \sum_{j=1}^{\left[\frac{t-s_{k}}{h}\right]}(-1)^{j+1} q C j\left(x(t-j h)-x_{k}\right) \\
& -\sum_{r=1}^{\left[\frac{t-s_{k}}{h}\right]}(-1)^{r+1} q C r\left(\left(x(t-r h)-x_{k}\right)\right) \|+L\left|\Lambda\left(h^{q}\right)\right| \sum_{r=1}^{\left[\frac{t-s_{k}}{h}\right.} q C r \\
& =L\left\|\left(\sum_{r=0}^{\left[\frac{t-s_{k}}{h}\right]}(-1)^{r+1} q C r\right)\left(\sum_{j=1}^{\left[\frac{t-s_{k}}{h}\right]}(-1)^{r+1} q C j\left(x(t-j h)-x_{k}\right)\right)\right\| \\
& +L\left|\Lambda\left(h^{q}\right)\right| \sum_{r=1}^{\left[\frac{t-s_{k}}{h}\right]} q C r .
\end{aligned}
$$

Substitute inequality (27) in (25), divide both sides by $h^{q}$, take the limit as $h \rightarrow 0^{+}$, use $\sum_{r=0}^{\infty} q C r z^{r}=(1+z)^{q}$ if $|z| \leq 1$ and condition 2(ii) and get

$$
\begin{aligned}
& { }_{s_{k}}^{c} D_{+}^{q} v(t) \leq{ }^{c} D_{+}^{q} V\left(t, x ; s_{k}\right)+L \lim _{h \rightarrow 0+} \frac{\Lambda\left(h^{q}\right)}{h^{q}} \lim _{h \rightarrow 0+}\left[\frac{t-s_{k}}{h} \sum_{r=1}^{h} q C r\right. \\
& \quad+L \lim _{h \rightarrow 0^{+}} \sup \left\|\left(\sum_{r=0}^{h}(-1)^{r+1} q C r\right)\left(\frac{1}{h^{q}} \sum_{j=1}^{h} q C j\left(x(t-j h)-x_{k}\right)\right)\right\| \\
& \left.={ }^{c} D_{+}^{q} V\left(t, x ; s_{k}\right) \leq-m V(t, x(t))\right)=-m v(t), t \in\left(s_{k}, T_{k+1}\right] .
\end{aligned}
$$

From (24) and (28) it follows the function $v(t)$ satisfies

$$
\begin{aligned}
& { }_{T_{0}}^{c} D_{+}^{q} v(t) \leq-m v(t) \quad \text { for } s_{k}<t \leq T_{k+1}, k=1,2, \ldots, \\
& v\left(T_{k}+\right) \leq C_{k} v\left(T_{k}\right), \quad \text { for } T_{k}<t \leq s_{k}, k=1,2, \ldots, \\
& v\left(T_{0}\right)=V\left(T_{0}, x_{0}\right) .
\end{aligned}
$$

Consider the solution of the IVP for RNIFrDE corresponding to (29) (with equalities instead of inequalities) and with a solution $u\left(t ; T_{0}, x_{0},\left\{T_{k}\right\}\right), t \geq T_{0}$. Therefore, the inequality 
$v(t)=V\left(t, x\left(t ; T_{0}, x_{0},\left\{T_{k}\right\}\right)\right) \leq u(t)=u\left(t ; T_{0}, V\left(T_{0}, x_{0}\right),\left\{T_{k}\right\}\right)$ holds and $V\left(t, x\left(t ; T_{0}, x_{0},\left\{\tau_{k}\right\}\right)\right) \leq$ $u\left(t ; T_{0}, V\left(T_{0}, x_{0}\right),\left\{\tau_{k}\right\}\right)$.

From Inequality (13), condition 2(i) of Theorem 1 and Lemma 4 for the function $u\left(t ; T_{0}, x_{0},\left\{\tau_{k}\right\}\right)$ we obtain for $t \geq T_{0}$ the inequalities

$$
\begin{aligned}
& E\left(\left\|x\left(t ; T_{0}, x_{0},\left\{\tau_{k}\right\}\right)\right\|^{p}\right)=\frac{1}{a} E\left(a\left\|x\left(t ; T_{0}, x_{0},\left\{\tau_{k}\right\}\right)\right\|^{p}\right) \\
& \leq \frac{1}{a} E\left(V\left(t, x\left(t ; T_{0}, x_{0},\left\{\tau_{k}\right\}\right)\right) \leq \frac{1}{a} E\left(u\left(t ; T_{0}, V\left(T_{0}, x_{0}\right),\left\{\tau_{k}\right\}\right)\right)\right. \\
& \leq \frac{1}{a} V\left(T_{0}, x_{0}\right) e^{-(\lambda-\mu)\left(t-T_{0}\right)} \leq \frac{b}{a}\left\|x_{0}\right\|^{p} e^{-(\lambda-\mu)\left(t-T_{0}\right)} .
\end{aligned}
$$

Inequality (30) proves the $p$-moment exponential stability.

Remark 3. If the condition 2(ii) of Theorem 1 is satisfied for $t>T+\Theta$ where the constant $\Theta=\Theta(T)>0$ then the zero solution is eventually p-moment exponentially stable.

Example 1. Let $d_{0}=0, d_{k}=\frac{1}{3^{k-1}}, k=1,2,3, \ldots$ be a given numbers. Then the condition (H2) is satisfied with $B=1.5$.

Consider the IVP for RNIFrDE

$$
\begin{aligned}
& { }_{0}^{c} D^{0.5} x(t)=-0.5 \frac{t}{\Gamma(0.5)}(x+y) \\
& { }_{0}^{c} D^{0.5} y(t)=0.5 \frac{t}{\Gamma(0.5)}(x-y) \quad \text { for } t \geq 0, \quad \xi_{k}+d_{k}<t<\xi_{k+1}, \\
& x(t)=0.5 \sin (t) x\left(\xi_{k}-0\right), \quad y(t)=\frac{0.5 t}{t+1} y\left(\xi_{k}-0\right) \quad \text { for } \xi_{k}<t<\xi_{k}+d_{k}, k=1,2, \ldots, \\
& x(0)=x_{0}, \quad y(0)=y_{0},
\end{aligned}
$$

where $x, y \in \mathbb{R}$, random variables $\xi_{k}$ are defined by $(4)$.

Let $V(x, y)=x^{2}+y^{2}$. The condition 3(i) of Theorem 1 is satisfied for $p=2$. The condition 3(iii) is reduced to

$$
V\left(t, I_{k}(t, x)\right)=(0.5 \sin (t) x)^{2}+\left(\frac{0.5 t}{t+1} y\right)^{2} \leq 0.25\left(x^{2}+y^{2}\right)=w_{k}(t) V(t, x),
$$

where $w_{k}(t) \equiv 0.25$. 
Let $T \in \mathbb{R}_{+}$be an arbitrary. Then using (21) we get

$$
\begin{aligned}
{ }^{c} D_{+}^{0.5} V(t, x ; T) & =\limsup _{h \rightarrow 0^{+}} \frac{1}{h^{0.5}}\left\{\left(x^{2}+y^{2}\right)-\sum_{r=1}^{\left[\frac{t-T}{h}\right]}(-1)^{r} 0.5 C r\left[\left(x-h^{0.5} f(t, x, y)\right)^{2}+\left(y-h^{0.5} g(t, x, y)\right)^{2}\right]\right\} \\
= & \left(x^{2}+y^{2}\right) \limsup _{h \rightarrow 0^{+}} \frac{1}{h^{0.5}} \sum_{r=0}^{\left.\frac{t-T}{h}\right]}(-1)^{r} 0.5 C r \\
& \left.+\limsup _{h \rightarrow 0^{+}} \frac{1}{h^{0.5}} \sum_{r=1}^{\left.\frac{t-T}{h}\right]}(-1)^{r} 0.5 C r\left[\left(x-h^{0.5} f(t, x, y)\right)^{2}+\left(y-h^{0.5} g(t, x, y)\right)^{2}-\left(x^{2}+y^{2}\right)\right]\right\} \\
= & \frac{\left(x^{2}+y^{2}\right)}{(t-T)^{0.5} \Gamma(0.5)}-(2 x f(t, x, y)+2 y g(t, x, y)) \limsup _{h \rightarrow 0^{+}} \sum_{r=1}^{h}(-1)^{r} 0.5 C r \\
+ & \left((f(t, x, y))^{2}+(g(t, x, y))^{2}\right) \limsup _{h \rightarrow 0^{+}} h^{0.5}\left[\frac{t-T}{h}\right] \\
= & \frac{\left(x^{2}+y^{2}\right)}{(t-T)^{0.5} \Gamma(0.5)}+(2 x f(t, x, y)+2 y g(t, x, y)) \\
= & \left(x^{2}+y^{2}\right)\left(\frac{1}{(t-T)^{0.5} \Gamma(0.5)}-\frac{t}{\Gamma(0.5)}\right) \\
= & \frac{V(x, y)}{\Gamma(0.5)}\left(\frac{1}{(t-T)^{0.5}}-t\right) .
\end{aligned}
$$

Note that for any $T>0$ there exists $\Theta>0$ such that $\frac{1}{(t-T)^{0.5}}-t<-1$ for $t \geq T+\Theta$. Therefore, the condition 2(iii) of Theorem 1 is satisfied with $m=\frac{1}{\Gamma(0.5)}$ for $t>T+\Theta$.

Also,

$$
E_{0.5}\left(-a_{0}\left(t-T_{0}\right)^{0.5}\right)\left(\lambda\left(t-T_{0}\right)\right) \leq\left(\lambda\left(t-T_{0}\right)\right) \leq M_{0} e^{\mu_{0}\left(t-T_{0}\right)}, \quad t \geq T_{0},
$$

with $M_{0}=1, \mu=0.5 \lambda$ and

$$
\begin{aligned}
& \frac{B\left(\lambda\left(t-T_{0}\right)\right)^{\alpha_{k+1}}+1}{\lambda\left(t-T_{0}-\sum_{i=1}^{k} d_{i}\right) \Gamma\left(\sum_{m=1}^{k} \alpha_{m}\right)} \prod_{i=1}^{k}\left|b_{i} e^{\lambda d_{i}}\left(\lambda\left(t-T_{0}\right)\right)^{\alpha_{i}}\right| \\
& =\frac{B\left(\lambda\left(t-T_{0}\right)\right)^{2}+1}{\lambda\left(t-T_{0}-\sum_{i=1}^{k} \frac{1}{3^{i-1}}\right) \Gamma\left(\sum_{m=1}^{k} \alpha_{m}\right)} \prod_{i=1}^{k} \mid b_{i} e^{\frac{\lambda}{3^{i-1}}\left(\lambda\left(t-T_{0}\right)\right)^{2} \mid} \\
& =\frac{B\left(\lambda\left(t-T_{0}\right)\right)^{2}+1}{\lambda\left(t-T_{0}-1.5\right) \Gamma(2 k)}\left(\lambda\left(t-T_{0}\right)\right)^{2} e^{1.5 \lambda}(0.25)^{k}\left(\frac{1}{\Gamma(0.5)}\right)^{k}
\end{aligned}
$$

and there exist $M_{k}, \mu_{k}>0$ such that the Inequality (11) holds for $t \geq T_{0}$. Therefore, the condition 3 of Theorem 1 is satisfied.

According to Remark 3 the zero solution is eventually mean square exponentially stable (eventually 2-moment exponentially stable).

\section{Conclusions}

The $p$-moment stability of the nonlinear Caputo fractional differential equations with impulses starting abruptly at some randomly distributed points and their action continue on intervals with 
given finite lengths. The type of the distribution of the random variables determining the moments of the impulses is very important. We study the case of Erlang distributed random variables between two consecutive moments of impulses. The stability is studied without the restriction about the constant length of the randomly occurred intervals of impulses. This study combines the ones of the theory of fractional differential equations and the probability theory. The study by base on the employing Lyapunov functions. The fractional Dini derivatives are applied to obtain some sufficient conditions. Since the exponential distribution is a partial case of Erlang distribution, the results in this paper are a generalization of those in Reference [17].

Author Contributions: Conceptualization, S.H.; methodology, S.H. and K.I.; formal analysis, S.H. and K.I.; investigation, S.H. and K.I.; writing-review and editing, S.H. and K.I.

Funding: This research received no external funding.

Acknowledgments: Research was partially supported by the Fund NPD, Plovdiv University, No. MU19-FMI-002 and No. FP19-FMI-002.

Conflicts of Interest: The authors declare no conflict of interest.

\section{References}

1. Bagley, R.L.; Calico, R.A. Fractional order state equations for the control of viscoelasticallydamped structures. J. Guid. Control Dyn. 1991, 14, 304-311. [CrossRef]

2. Laskin, N. Fractional market dynamics. Phys. A Stat. Mech. Its Appl. 2000, 287, 482-492. [CrossRef]

3. Molina-Garcia, D.; Sandev, T.; Safdari, H.; Pagnini, G.; Chechkin, A.; Metzler, R. Crossover from anomalous to normal diffusion: Truncated power-law noise correlations and applications to dynamics in lipid bilayers. New J. Phys. 2018, 20, 103027. [CrossRef]

4. Dos Santos. M.A. Fractional Prabhakar Derivative in Diffusion Equation with Non-Static Stochastic Resetting. Physics 2019, 1, 40-58. [CrossRef]

5. Church, K.E.M.; Smith, R.J. Existence and uniqueness of solutions of general impulsive extension equations with specification to linear equations. Dyn. Cont. Discr. Impuls. Syst. Ser. B Appl. Algorithms 2015, 22, 163-197.

6. Das, S.; Pandey, D.N.; Sukavanam, N. Existence of solution of impulsive second order neutral integrodifferential equations with state delay. J. Integral Equ. Appl. 2015, 27, 489-520.

7. Hernandez, E.; Pierri, M.; O'Regan, D. On abstract differential equations with non instantaneous impulses. Topol. Methods Nonlinear Anal. 2015, 46, 1067-1088.

8. Kumar, P.; Pandey, D.N.; Bahuguna, D. On a new class of abstract impulsive functional differential equations of fractional order. J. Nonlinear Sci. Appl. 2014, 7, 102-114. [CrossRef]

9. $\mathrm{Li}, \mathrm{P} . ; \mathrm{Xu}, \mathrm{C}$. Boundary value problems of fractional order differential equation with integral boundary conditions and not instantaneous impulses. J. Funct. Spaces 2015, 2015, 954925. [CrossRef]

10. Liao, Y.M.; Wang, J.R. A note on stability of impulsive differential equations. Bound. Value Probl. 2014, 2014, 67. [CrossRef]

11. Pierri, M.; Henriquez, H.R.; Prokopczyk, A. Global solutions for abstract differential equations with non-instantaneous impulses. Med. J. Math. 2016, 13, 1685-1708. [CrossRef]

12. Boudaoui, A.; Caraballo, T.; Ouahab, A. Stochastic differential equations with non-instantaneous impulses driven by a fractional Brownian motion. Discr. Cont. Dyn. Syst. B 2017, 22, 2521-2541. [CrossRef]

13. Sanz-Serna, J.M.; Stuart, A.M. Ergodicity of dissipative differential equations subject to random impulses. J. Differ. Equ. 1999, 155, 262-284. [CrossRef]

14. Wu, S.; Hang, D.; Meng, X. p-Moment Stability of Stochastic Equations with Jumps. Appl. Math. Comput. 2004, 152, 505-519. [CrossRef]

15. Wu, H.; Sun, J. $p$-Moment Stability of Stochastic Differential Equations with Impulsive Jump and Markovian Switching. Automatica 2006, 42, 1753-1759. [CrossRef]

16. Yang, J.; Zhong, S.; Luo, W. Mean square stability analysis of impulsive stochastic differential equations with delays. J. Comput. Appl. Math. 2008, 216, 474-483. [CrossRef] 
17. Agarwal, R.; Hristova, S.; O’Regan, D.; Kopanov, P. Differential equations with random Gamma distributed moments of non-instantaneous impulses and $p$-moment exponential stability. Demonstr. Math. 2018, 51, 151-170. [CrossRef]

18. Agarwal, R.; Hristova, S.; O’Regan, D.; Kopanov, P. p-moment exponential stability of differential equations with random noninstantaneous impulses and the Erlang distribution. Int. J. Pure Appl. Math. 2016, 109, 9-28. [CrossRef]

19. Chen, Y.; Wang, J.R. Continuous Dependence of Solutions of Integer and Fractional Order Non-Instantaneous Impulsive Equations with Random Impulsive and Junction Points. Mathematics 2019, 7, 331. [CrossRef]

20. Liu, S.; Wang, J.R.; Shen, D.; O'Regan, D. Iterative learning control for noninstantaneous impulsive fractional-order systems with varying trial lengths. Int. J. Robust Nonlinear Control 2018, 28, 6202-6238. [CrossRef]

21. Yang, D.; Wang, J.R. Non-instantaneous impulsive fractional order implicit differential equations with random effects. Stoch. Anal. Appl. 2017, 35, 719-741. [CrossRef]

22. Das, S. Functional Fractional Calculus; Springer: Berlin/Heidelberg, Germany, 2011.

23. Diethelm, K. The Analysis of Fractional Differential Equations; Springer: Berlin/Heidelberg, Germany, 2010.

24. Anguraj, A.; Vinodkumar, A.; Malar, K. Existence and stability results for random impulsive fractional pantograph equations. Filomat 2016, 30, 3839-3854. [CrossRef]

25. Anguraj, A.; Ranjini, M.C.; Rivero, M.; Trujillo, J.J. Existence results for fractional neutral functional differential equations with random impulses. Mathematics 2015, 3, 16-28. [CrossRef]

26. Vinodkumara, A.; Indhumathib, P. Global existence and stability results for mild solutions of random impulsive partial integro-differential equations. Filomat 2018, 32, 439-455. [CrossRef]

27. Zhang, S.; Jiang, W. The existence and exponential stability of random impulsive fractional differential equations. Adv. Differ. Equ. 2018, 2018, 404. [CrossRef]

28. Iwankiewicz, R. Dynamical Mechanical Systems Under Random Impulses; World Scientific Publishing: London, UK, 1995; Volume 36.

29. Devi, J.V.; Rae, F.A.M.; Drici, Z. Variational Lyapunov method for fractional differential equations. Comput. Math. Appl. 2012, 64, 2982-2989. [CrossRef]

30. Ahmad, B.; Sivasundaram, S. Existence results for nonlinear impulsive hybrid boundary value problems involving fractional differential equations. Nonlinear Anal. Hybrid Syst. 2009, 3, 251-258. [CrossRef]

31. Benchohra, M.; Slimani, B.A. Existence and uniqueness of solutions to impulsive fractional differential equations. Electron. J. Differ. Equ. 2009, 2009, 1-11.

32. Wang, G.; Ahmad, B.; Zhang, L.; Nieto, J. Comments on the concept of existence of solution for impulsive fractional differential equations. Commun. Nonlinear Sci. Numer. Simul. 2014, 19, 401-403. [CrossRef]

33. Agarwal, R.; Hristova, S.; O'Regan, D. p-Moment exponential stability of Caputo fractional differential equations with noninstantaneous random impulses. J. Appl. Math. Comput. 2017, 55, 149-174.

(C) 2019 by the authors. Licensee MDPI, Basel, Switzerland. This article is an open access article distributed under the terms and conditions of the Creative Commons Attribution (CC BY) license (http:/ / creativecommons.org/licenses/by/4.0/). 